Discussion Paper No. 822

\title{
SMOKERS, SMOKING DEPRIVATION, AND TIME DISCOUNTING
}

\author{
Shoko Yamane \\ Hiroyasu Yoneda \\ Taiki Takahashi \\ Yoshio Kamijo \\ Yasuhiro Komori \\ Fumihiko Hiruma \\ Yoshiro Tsutsui
}

November 2011

The Institute of Social and Economic Research

Osaka University

6-1 Mihogaoka, Ibaraki, Osaka 567-0047, Japan 


\title{
Smokers, Smoking Deprivation, and Time Discounting*
}

\author{
Shoko Yamane ${ }^{\mathrm{A}}$ \\ Hiroyasu Yoneda ${ }^{\mathrm{B}}$ \\ Taiki Takahashi ${ }^{\mathrm{C}}$ \\ Yoshio Kamijo $^{\text {D }}$ \\ Yasuhiro Komori ${ }^{\mathrm{E}}$ \\ Fumihiko Hiruma $^{\mathrm{F}}$ \\ Yoshiro Tsutsui $^{\mathrm{G}}$
}

\footnotetext{
* We gratefully acknowledge insightful comments by Noah Smith.

A Shoko Yamane, MA; Graduate School of Economics, Osaka University; 1-7, Machikaneyama, Toyonaka 560-0043 Japan; Phone +8190-1950-3909 ;

e-mail hgp011ys@mail2.econ.osaka-u.ac.jp

${ }^{\text {B }}$ Hiroyasu Yoneda, Ph.D.; Institute of Social and Economic Research, Osaka University; 6-1 Mihogaoka, Ibaraki, Osaka 567-0047; phone +81-6-6879-8552;

fax +81-6-6879-8584; e-mail yoneda-h@econ.osaka-u.ac.jp

${ }^{\mathrm{C}}$ Taiki Takahashi, Ph.D.; Center for Experimental Research in Social Sciences Department of Behavioral Science, Hokkaido University; N.10, W.7, Kita-ku, Sapporo, 060-0810 Japan; phone +81-11-706-3057; fax +81-11-706-3066;

e-mail ttakahashi@lynx.let.hokudai.ac.jp

D Yoshio Kamijo, Ph.D.; Waseda Institute for Advanced Study, Waseda University; 1-6-1 Nishiwaseda, Shinjukuku, Tokyo 169-8050 Japan; phone +81-3-5286-2103; e-mail yoshio.kamijo@gmail.com

E Yasuhiro Komori, MA; 5-43-4 Minamisenju, Arakawaku, Tokyo 116-0003 Japan, phone +81-3-3801-6002; e-mail yasuhiro.komori@gmail.com

F Fumihiko Hiruma, MA; School of Commerce, Waseda University; 1-6-1 Nishiwaseda Shinjuku-ku Tokyo 169-8050 Japan; phone +81-3-5286-2014; e-mail hiruma@waseda.jp

G Corresponding author, Yoshiro Tsutsui, Ph.D.; Graduate School of Economics, Osaka University; 1-7, Machikaneyama, Toyonaka 560-0043 Japan; Phone +81-6-6850-5223; Fax +81-6850-5274; e-mail tsutsui@econ.osaka-u.ac.jp
} 


\begin{abstract}
This paper investigates whether smokers exhibit greater time discounting than non-smokers, and how short-term nicotine deprivation affects time discounting. A unique feature of our experiment is that our subjects receive rewards not only of money, but also of actual tobacco. This is done in order to elicit smokers' true preferences. Smokers are more impatient than non-smokers, consistent with previous studies. Additionally, nicotine deprivation makes smokers even more impatient. These results suggest that nicotine concentration has different effects on time preferences in the short and long runs.
\end{abstract}

JEL Classifications: D03, D90, I10, Q57

Keywords: time discounting, nicotine concentration, smoking deprivation, panel logit analysis, economic experiment 


\section{Introduction}

It is well-established that smokers are more impatient than non-smokers (Brick et al. 1999, Mitchell 1999, Baker et al. 2003, Ohmura et al. 2005, Reynolds 2004, Reynolds et al. 2004, 2007). ${ }^{1}$ Experiments using animals suggest that this is due to a chronic (but not acute) increase in nicotine concentration (Dallery and Locey 2005, Tsutsui-Kimura et al. 2010). ${ }^{2}$

The purpose of this study is twofold. We first seek to confirm the result that smokers display greater time discounting than non-smokers. We then investigate the short-term effects of nicotine deprivation on time discounting.

If time discounting simply corresponds to the concentration of nicotine in a person's body, a short-term deprivation of nicotine should reduce a person's time discount rate, by reducing this concentration. Indeed, Dallery and Locey (2005) report that an increase in impulsiveness induced by chronic nicotine administration is reversible in rats. However, casual observation suggests that smokers become more irritated and impatient when they abstain from smoking for a while. Several studies support this intuition. An experiment by Sayette et al. (2005) finds that the urge to

\footnotetext{
${ }^{1}$ However, Khwaja et al. (2007), based on survey results, report that there are no significant differences in revealed rates of time discounting between smokers and non-smokers.

${ }^{2}$ Note that there exists reverse causality, in that the time discount rate significantly affects an individual's decision to start smoking (Sato and Ohkusa, 2003).
} 
smoke may affect time perception, and that smokers who crave nicotine overpredict the duration and intensity of their own future smoking urges. Using opioids rather than tobacco, Badger et al. (2007) find that heroin addicts value an extra dose of the heroin substitute Buprenorphine more highly when they are currently craving than when they are currently satiated. Similarly, Giordano et al. (2002) find that the degree of discounting was significantly higher when subjects are opioid-deprived, and conclude that opioid deprivation increases the degree to which dependent individuals discount delayed heroin and money. These studies suggest a negative rather than a positive relationship between time discounting and the concentration of a drug in an addict's body.

Examining the relation between nicotine deprivation and impatience, Mitchell (2004) and Field et al. (2006) report that deprivation makes subjects more impulsive. However, comparing one-day and 14-day abstinence groups, Yoon et al. (2009) find no significant difference in time discounting tasks. Using a three-hour deprivation period, Dallery and Raiff (2007) report no significant differences in time discounting between active nicotine patch and placebo patch groups. Although these results are not conclusive, they suggest that the long-term and short-term effects of nicotine deprivation on impatience may differ. To resolve this puzzle, we propose that when a 
non-smoker starts smoking, the long-term increase in nicotine concentration makes her more impatient in general, but a decrease in nicotine concentration due to a brief cessation of smoking makes her even more impatient for the duration of deprivation. ${ }^{3}$

To explore both the long- and short-term effects of nicotine addiction, we conduct an experiment comparing time discounting between smokers and non-smokers, as well as between deprived smokers and non-deprived smokers. The salient difference of our experiments from previous studies such as Mitchell (2004) and Field et al. (2006) is that our subjects are asked to choose between receiving nicotine earlier and receiving nicotine later. In contrast, Mitchell (2004) asks her subjects to choose between receiving a number of cigarettes (up to 60 ) and US\$10 immediately, or receiving a larger amount of money in the future (up to 365 days). This task does not give subjects the opportunity to choose the time at which they will be allowed to smoke. In addition, the 60 cigarettes are not all smoked at the time they are received, leaving some ambiguity in the timing of the nicotine receipt. Thus, subjects facing this cigarette-money tradeoff should show

\footnotetext{
${ }^{3}$ Several studies investigate what kinds of people more easily abstain from smoking. For example, Krishnan-Sarin et al. (2007) study thirty adolescent smokers, who participated in a high school based smoking cessation program; sixteen participants (53\%) were abstinent from smoking at the completion of the four-week study. Compared to abstinent adolescents, those not achieving abstinence discounted monetary rewards more. Thus, it may be the case that more impulsive adolescents were unable to achieve abstinence. Dallery and Raiff (2007) report that those who had higher time discounting tended to choose smoking more often than money, suggesting that they had more difficulty abstaining. Conducting a five-month follow-up survey of 608 Japanese adults who had just begun smoking cessation, Ida et al. (2011) found that cessation successes are more risk averse than cessation failures, and that time preference rates decrease for cessation successes and increase for cessation failures.
} 
the same rate of time preference as if both alternatives were purely monetary (since they presumably assign a fixed monetary value to the immediate receipt of 60 cigarettes). Surprisingly, however, Mitchell finds that nicotine-deprived subjects become more impulsive in a cigarette-money session, but not in a money-money session, suggesting that the framing of the choice has some impact on the intertemporal decisions of her subjects.

Field et al. (2006) ask subjects in their money-money task to choose between fixed amounts of money (£500) received later vs. some amount of money received immediately. The delay is set at either 1 week, 2 weeks, 1 month, 6 months, 1 year, 5 years, or 25 years. The subjects in their cigarette-cigarette task are asked to choose between amounts of cigarettes that correspond to the monetary rewards in the money-money task. ${ }^{4}$ They find that nicotine-deprived participants show more pronounced delay discounting in both tasks. However, our critique of Mitchell (2004) applies to Field et al. (2006) as well; we doubt that either the "cigarette-money task” in Mitchell (2004) or the "cigarette-cigarette task" in Field et al. (2006) is the best way to elicit preferences on smoking. In our experiment, in contrast, subjects choose both the amount and the timing of their smoking reward. Our experiment is unique in that we

\footnotetext{
${ }^{4} £ 500$ corresponds to 100 packs, where one pack contains 20 cigarettes. The rewards are hypothetical in Field et al. (2006), and are not actually paid to the subjects.
} 
pay actual rewards not only in the "money session," but also in the "tobacco session"; at the end of the experiment, subjects actually smoke according to their choices earlier in the session. ${ }^{5}$ We do this because we believe that precision in the specification of incentives is crucial to the accurate elicitation of preferences, especially in the case of smoking.

It is known that people have different discount rates for different consumed goods; these differences are called "domain effects" (Frederick et al., 2002; Odum and Baumann, 2007). In our case, deprived smokers may be highly impatient with regards to tobacco, but more patient with regards to other goods such as money.

The rest of the paper proceeds as follows. In the next section, we explain our experimental design. In section 3, we present the preliminary results of the experiments. In section 4, we explain the main results of our panel logit estimation. Section 5 concludes.

\section{Experimental design}

\subsection{Basic setup}

Our subjects consist of three groups: non-smokers, smokers who smoked as usual on the

\footnotetext{
${ }^{5}$ Yoon et al. (2009) conduct a choice task involving real money and cigarettes; however, subjects are requested to choose between one puff now and $\$ 0.25$ now, so that their task is not an intertemporal choice.
} 
day of the experiment, and smokers who were deprived of smoking for 12 hours before the beginning of the experiment. Subjects who comprised the "usual smoker group" on the first day became members of the "deprived smoker group" on the second day, and vice versa. $^{6}$

Subjects were requested to choose one of two options, A or B, displayed on a computer in front of each subject. ${ }^{7}$ Those who chose option A received a smaller reward earlier, and those who chose option B received a larger reward later.

We varied four variables over our treatment groups: 1) the size of the reward in option A, 2) the "delay," 3) the "interval," and 4) the "rate of return.” The "delay” is defined as the difference between the time at which the option is chosen $(t=0)$ and the time at which option A is received. The "interval" is defined as the difference between the times at which options A and B are received. The "rate of return" is defined as the amount of reward in option B minus the amount in option A, divided by the interval.

\subsection{Hypothetical tobacco, money, and real tobacco sessions}

The experiment consisted of three sessions: the "hypothetical tobacco," "money," and "real tobacco" sessions.

\footnotetext{
${ }^{6}$ Using the same subjects in the two sessions enabled within-subjects comparisons between deprived and non-deprived conditions.

7 The experiment was carried out using the software Hot Soup Play.
} 


\section{Real tobacco session:}

We begin with the explanation of the real tobacco session, as this is, to our knowledge, the first time such a choice has been offered to experimental subjects. The rationale behind using real tobacco is that the desire to smoke is an instinctive rather than a rational motivation, so that the belief that rewards will actually be paid (i.e. that subjects will be able to smoke) is necessary to elicit true preferences. Thus, we set up the experiment so that each subject would smoke a specified amount at the exact time specified in the option she selected.

We used five values for the delay; 32, 29, 26, 23, and 20 minutes. The interval was fixed at 30 minutes. The real tobacco session was divided into five blocks; in each block, 16 questions were asked. Subjects had 3 minutes to answer the 16 questions in a block. The delay was fixed in each block: e.g. 32 minutes in the first block, 29 minutes in the second block and so on. Therefore, those who chose the earlier option in a particular "tobacco" question all smoked at the same time, i.e. 20 minutes from the time the last question was answered. Those who chose the later option smoked 50 minutes from the time the last question was answered, since the interval was set at 30 minutes.

The tobacco reward took one of six possible values: one puff, two puffs, a half cigarette, one cigarette, one and a half cigarettes, and two cigarettes. After some 
preliminary trials, it was decided that eight puffs was equivalent to smoking one cigarette.

Ten rates of return were used; 0, 33.3, 50, 100, 200, 300, 500, 700, 1100, and 1500

(\%) for a 30-minute interval. Using these parameter values, 16 questions were asked for each delay, so that 80 questions were asked in total in the real tobacco session (Table 1).

\section{Hypothetical tobacco session:}

Although our real tobacco session was able to elicit the subjects' preferences over the given time horizon of 50 minutes, longer time periods could not be explored. To ask questions concerning longer delays and intervals, we added a "hypothetical tobacco session," in which subjects did not actually smoke at the end of the experiment.

In this session, the hypothetical smoking rewards were the same as those in the real tobacco session. The five delays were zero, one hour, three hours, 12 hours, and 24 hours. The interval was fixed at 12 hours. The ratios of the rates of return were the same as those of the real tobacco session, relative to the interval. Based on these conditions, 16 questions were asked for each delay, so that 80 questions were asked in total in the hypothetical tobacco session (Table 1).

\section{Money session:}


The money session had three possible rewards; 1000, 2000, and 3000 yen. ${ }^{8}$ Five delays were considered: today, one week, two weeks, three weeks, and four weeks. The interval was fixed at two weeks. Six different annualized rates of returns were chosen: 0 , 50, 100, 150, 200, and 300 (\%). Based on these conditions, 16 questions were asked for each delay, so that a total of 80 questions were asked in the money session (Table 2).

At the end of the experiment, one question was randomly selected out of 80 questions for the money and real tobacco sessions respectively, and subjects received a reward (both money and smoking), based on their choice in the selected question, at the time stated in the chosen option. Smokers in both the "usual” and "deprived" smoking groups earned an average of $¥ 4,450$ for two days, and non-smokers, who attended only the money session on one day, earned $¥ 1,923$. In addition, smokers and non-smokers were paid $¥ 6,666$ (for two days) and ¥2,222, respectively, in cash as compensation for participation, so that total per-capita rewards were $¥ 11,116$ for smokers and $¥ 4,145$ for non-smokers.

\subsection{Flow of the experiment}

After the instructions were read, the hypothetical tobacco session, money session, and

\footnotetext{
${ }^{8}$ At this time the exchange rate was about $\$ 1=¥ 90$ or $¥ 91$.
} 
real tobacco sessions were conducted in that order. Only the usual smoker group was allowed to smoke during the breaks between the sessions. The real tobacco session was divided into five blocks, each of which involved 16 questions in three minutes. After each real tobacco session finished, one of the 80 questions was randomly selected, and each subject smoked the amount of tobacco at the time designated in her chosen option in the selected question. During this 50-minute smoking time, subjects answered a questionnaire and were paid the show-up fee. ${ }^{9}$ After all the subjects smoked, they waited for 30 minutes in the laboratory, during which time they were allowed to do anything other than smoke (if applicable). This 30-minute prohibition of smoking was announced in the instructions at the beginning of the experiment, before subjects made their choices. This was done to assure that subjects did not smoke on their own immediately after leaving the experiment, since this opportunity would distort their intertemporal choice. $^{10}$

\subsection{Implementation of the experiment}

The experiment was conducted on January 12-14 (first wave) and February 20-21

\footnotetext{
${ }^{9}$ Most of the rewards in the money session, except for the ones received immediately, were paid later at the specified times by bank transfer.

${ }^{10}$ In the hypothetical tobacco session, we asked the subjects to "suppose you were unable to smoke for 24 hours after the experiment" when they made their choices.
} 
(second wave), 2010 at Osaka University, Japan. The subjects consisted of 50 smokers (male=49, female=1) and 17 non-smokers (male=13, female=4). ${ }^{11}$ All of the non-smokers were in the first wave. Of the smokers, 14 subjects (all male) were in the first wave and the rest of the subjects, 36 (male=35, female=1) were in the second wave of the experiment.

3. Preliminary results

\subsection{Compliance with the no-smoking requirement}

We asked the subjects of the deprived smoker group to stop smoking 12 hours prior to the beginning of the experiment. ${ }^{12}$ In order to verify that this was done, we gave these subjects a breath test and checked the CO concentration of their exhalations, using a “smokerlyzer” tool produced by Bedfont Scientific Ltd. The tool provides two measures of the likelihood of recent smoking; PPM (Parts Per Million) of CO in the lungs, and \%COHb (percent of carboxyhemoglobin) in the blood.

The mean of PPM among the deprived smokers was 3.24, while that of the usual smokers was 8.20, so that the deprived smokers showed significantly lower PPM (t(98) $=4.74, \mathrm{p}<0.000)$. The deprived smokers had $1.04 \% \mathrm{COHb}$ on average, while the usual

${ }^{11}$ Mitchel (2004) uses only eleven smokers. Field et al. (2006) use 30 smokers.

12 The experiments began at either 10 am or $1 \mathrm{pm}$. 
smokers had 1.86, so that again the deprived smokers showed significantly lower smoking activity by this measure $(\mathrm{t}(98)=4.53, \mathrm{p}<0.000)$. These results indicate that the 12-hour injunction against smoking was generally obeyed. Inspection of the individual records revealed that all the subjects who showed high nicotine concentrations under the usual smoking condition show a large decline in concentration levels when deprived.

\subsection{Effectiveness of the 12-hour nicotine deprivation period}

It is important that the 12-hour nicotine deprivation period be long enough to strengthen the subjects' desire to smoke. ${ }^{13}$ In order to verify this, we asked the following question four times during the experiment ((1) just after the start of the experiment, (2) just after the hypothetical tobacco session, (3) just after the money session, and (4) just after the real tobacco session):

Question: How strongly do you want to smoke now? Please rate your desire from 1 (I do not want to smoke now) to 10 (I do want very much to smoke now).

The result is shown in Figure 1. The deprived group reported significantly higher desire to smoke than the usual smoker group ((1): $\mathrm{t}(98)=-6.16, \mathrm{p}=0.000,(2): \mathrm{t}(98)=$

${ }^{13}$ Mitchel (2004) asked her subjects to stop smoking for 24 hours; Field et al. (2006) 13 hours; and Dallery and Raiff (2007) 3 hours. 
$-4.97, \mathrm{p}=0.000,(3): \mathrm{t}(98)=-7.17, \mathrm{p}=0.000,(4): \mathrm{t}(98)=-7.10, \mathrm{p}=0.000)$. Also, while the smoking desire reported by the usual smoker group does not show an upward trend over the course of the experiment, that of the deprived group does. This is to be expected, because the usual smoker group is allowed to smoke during the breaks between the sessions, while the deprived group is not.

We also asked the subjects the following question at the end of the experiment.

Question: Suppose that you were not allowed to smoke at all for 24 hours, starting now. How much would you pay in order to smoke one cigarette now?

The average answer of usual smokers was ¥116.4, while that of deprived smokers was ¥210.8. Again these two groups’ smoking appetites differed during the experiment, although the difference is not statistically significant at the $5 \%$ level $(\mathrm{t}(70)=-1.67$, $\mathrm{p}=0.100)$.

\subsection{Average choices of the subject groups}

First, we report the number of rounds in which non-smokers, usual smokers, and deprived smokers, respectively, chose the later option. We code the choice as a dummy variable that equals 1 if option $B$ (the later option) is chosen, and 0 otherwise. The results are shown in Figure 2. The vertical axis in the figure gives the mean of this 
variable for each group.

From this figure it is apparent that non-smokers tend to choose the later option.

The difference in the mean between all smokers and non-smokers is significant $(\mathrm{t}$ $(9358)=8.792, \mathrm{p}=0.000)$.

Although the difference between deprived smokers and usual smokers is small in size, it is significant at the $5 \%$ level in the real tobacco session $(t(7998)=2.142$, $\mathrm{p}=0.032$ ). However, it is not significant in the hypothetical tobacco session $(\mathrm{t}(7998)=-0.631, \mathrm{p}=0.528)$ and “money session" $(\mathrm{t}(7998)=-0.761, \mathrm{p}=0.447)$. This simple analysis suggests that deprived smokers are more impatient than usual smokers when it comes to choices involving actual tobacco.

4. Panel logit analysis

\subsection{Estimation method}

To quantify time discounting for the three groups, we estimate a panel logit model, where the dependent variable is a choice dummy that takes a value of unity if a subject chooses a later option and zero if she chooses an earlier option. An alternative method would have been to first estimate separately the time discount rates for each treatment group specified by the delay, the interval, and the amount of rewards, and then to 
compare these. However, the method we use has the advantage of efficient use of all the information contained in the 240 total choices made by the subjects. More importantly, the two-step method assumes that each subject makes all decisions based only on her personal constant per-period time discount rate, an assumption that has been found questionable by a number of studies (Frederick et al. 2002, Kirby et al. 1995, Benzion et al. 1989, Loewenstein et al. 1992); our method requires no such assumption.

Other studies have adopted approaches that differ than ours. Previous studies such as Bickel et al. (1999), Reynolds (2004), Dallery and Raiff (2007), and Ohmura et al. (2005) estimate hyperbolic discounting functions. Field et al. (2006) use area under the curve (AUC), first proposed by Myerson et al. (2001), to measure time discounting. However, we have reasons for not using either of these approaches. Evaluation of the degree of hyperbolic discounting by estimation of the hyperbolic function is limited in that it assumes a specific functional form. AUC does not; however, calculating AUC for each subject at the first stage, and then comparing AUCs between smokers and non-smokers, sacrifices efficiency compared to the full-information method that we use.

It is believed that intertemporal choice is affected by the delay, the interval, and the magnitude of the reward (Kinari et al. 2009). Therefore, our explanatory variables are the rate of return $(R E T U R N)$, the delay (DELAY), and the amount of reward 
$\left(\right.$ AMOUNT) ${ }^{14}$ We also include a dummy variable for whether a subject is a smoker (D_SMOKER) for the analysis of smokers vs. non-smokers, i.e. the estimation using the data of the "money session." If the coefficient on this dummy is negative, it implies that smokers tend to choose later options, and are therefore less patient.

On the other hand, in the analysis of deprivation, the key variable measuring the effect of short-term nicotine deprivation is a dummy variable for deprived smokers (D_DEPRIVATION). All the data obtained in the three sessions are used for the analysis of deprivation. ${ }^{15}$ Explanations of all variables used in the regression analysis are given in the Appendix.

\subsection{Smokers vs. non-smokers}

The results of the estimation for smokers vs. non-smokers are presented in Table 3. The total number of observations is 5360. Only in the "money session" do we compare smokers with non-smokers. The left-most columns of the table show the coefficients on DELAY and AMOUNT. The coefficient on the smoker dummy is significantly negative, implying that smokers are more impatient than non-smokers. Although the coefficient

\footnotetext{
${ }^{14}$ The interval is fixed in each session, so that its effect is included in the constant term.

${ }^{15}$ Although smokers are also compared with non-smokers in the money session, only the results for deprived vs. non-deprived smokers are used in the analysis of deprivation.
} 
on $A M O U N T$ is significantly positive, implying that subjects become more patient for large rewards (the magnitude effect), the coefficient on the DELAY variable is not significant. Thus, we see no delay effect in this regression specification.

In the middle columns, the results for the delay and amount dummies are shown. The dummy variables representing whether the reward was given today (D_DELAY_M1, where " $M 1$ " represents the money session) and whether the amount was $¥ 1,000$ (D_AMOUNT_M1) are omitted for the benchmark specification. Again, the coefficient on D_SMOKER is significantly negative, confirming that smokers are more impatient. The amount dummies are significantly positive, and the coefficient on the dummy representing ¥3,000 (D_AMOUNT_M3) is larger than that for $¥ 2,000$ (D_AMOUNT_M2), confirming the existence of magnitude effects over the entire range of rewards. The delay dummies representing one and two weeks later (D_DELAY_M2 and $D \_D E L A Y \_M 3$ ) are significantly positive at the $1 \%$ level, while those for three and four weeks later (D_DELAY_M4 and D_DELAY_M5) are only significant at the 5\% level, with smaller point estimates, so that delay effect can only be unambiguously observed over periods of one or two weeks. ${ }^{16}$ This last result is consistent with Kinari et al. (2009) and Sasaki et al. (2011).

\footnotetext{
${ }^{16}$ This may be the reason why no delay effect is found when the delay variable itself is used as a regressor instead of these dummies.
} 
The right-hand columns of Table 3 show the results when dummies for the different rates of return are used as regressors instead of the return variable itself. The coefficients on the return dummies are significantly positive, and are larger for larger returns, confirming that subjects' choices were rational with respect to returns. The coefficients on the delay and amount dummies are similar in size to the corresponding coefficients in the previous regression.

\subsection{Effects of smoking deprivation}

In the upper panel of Table 4, we present the results for the effect of nicotine deprivation for all three sessions, using DELAY, AMOUNT, and RETURN as explanatory variables in addition to a dummy for the deprived smoker group. The smoking deprivation dummy is significant only for the real tobacco session. The coefficient on the dummy variable is negative, implying that smoking deprivation makes subjects more impatient. In the hypothetical tobacco and money sessions, the dummy variable is not significant. These results suggest that subjects reveal their true smoking preferences only when incentives are appropriate; i.e., when the smoking reward is real.

The coefficient on the delay differs over the three sessions. It is significantly negative in the real tobacco session, implying that the subjects become more impatient 
with respect to smoking as the delay becomes longer. This is the opposite of the typical delay effect. In the money session, the coefficient on the delay is significantly positive, showing the usual delay effect. In the hypothetical tobacco session, the coefficient is not significant.

The coefficient on the amount of reward is significantly positive in the money session, implying the usual magnitude effect; subjects become more patient when the amount of reward is large. However, in the real and hypothetical tobacco sessions, the effect is reversed; when the reward is larger, subjects are less patient.

The coefficient on the rate of return is positive in all sessions, indicating that subjects are rational with respect to rates of return.

When dummies for the delay and amount are used as regressors instead of the raw variables, the results are essentially unchanged (Table 4, lower panel). The smoking deprivation dummy is significant only in the real tobacco session. For the return variable, we do not use dummies; this is in order to avoid the dummy variable trap, as the return dummies are linearly dependent with the delay and amount dummies in the real and hypothetical tobacco sessions. The return variable is positive and significant in all sessions, as shown in the upper panel of the table. ${ }^{17}$

\footnotetext{
${ }^{17}$ For the money session, we also estimate an equation including all return dummies (the results
} 
The usual delay effect is only observed in the money session, as shown in the upper panel. However, we find that the delay effect only operates over one week. In the real and hypothetical tobacco sessions, the coefficients on the delay dummies are negative, implying that the opposite of the usual delay effect is found for some delays (shown in the upper panel).

For the magnitude effect, the coefficients of the amount dummies in the money session are positive and increasing in the amount, again confirming the usual magnitude effect. In the real and hypothetical tobacco sessions, the coefficients of the dummies on two puffs and half a cigarette are significantly positive, while those for larger amounts are significantly negative, suggesting that the amount of smoking has a nonlinear and complex effect on the choice.

In sum, nicotine deprivation makes subjects more impatient with regards to smoking, but not to money. The delays and amount of rewards matter for intertemporal choices, but differ depending on whether the choice is over tobacco or money. Thus, we find a domain effect for time discounting.

are omitted to save space). All the coefficients on the dummies are significantly positive and increasing in the amount. Thus, rationality of subjects' choices with respect to returns is again confirmed. The delay and magnitude effects are unchanged from those in the upper panel. 


\subsection{Robustness check}

Our subjects consist of both light and heavy smokers. Figure 3 presents a histogram of the number of cigarettes that the subjects smoke per day, which ranges from 1.5 to 25 cigarettes with a mode of ten cigarettes. In the previous subsection, we found that short-term deprivation makes subjects impatient in the real tobacco session. This tendency should be stronger for heavy smokers and weaker for light smokers. Thus, as a robustness check, we separate our 50 smokers into "heavy smoker" and "light smoker" groups, and measure the difference between the two.

It seems natural to separate the groups at the mode of ten cigarettes. In one specification (specification (a)), the light smoker group consists of those who smoke less than or equal to ten cigarettes (31 subjects) and the heavy smoker group consists of the other 17 subjects; in specification (b), the light smoker group consists of those who smoke less than ten cigarettes (17 subjects) and the heavy smoker group consists of the remaining 31 subjects. ${ }^{18}$

The results for specification (a) are presented in the upper panel of Table 5. In the real tobacco session, although the deprivation dummy is significantly negative for the

18 The number of observations is smaller than 50 because those who chose only A or only B are excluded. 
heavy smoker group, it is not significant for the light smoker group. ${ }^{19}$ The coefficient on the deprivation dummy for the heavy smoker group is larger in absolute value than for the whole sample.

In the lower panel, the results of separation (b) are presented. They are essentially the same, confirming our hypothesis. In addition, the heavy smoker group becomes significantly impatient when deprived, even in the hypothetical tobacco session. This robustness check strongly suggests that the results of the previous section have captured a real effect.

\section{Conclusions}

In this paper, we investigated whether smokers show higher time discounting than non-smokers, and how short-term nicotine deprivation affects time discounting. A unique feature of our experiment is to offer subjects a choice between two smoking options, and to give rewards to subjects according to their choices not only in the money session, but also in the tobacco session, in order to measure domain effects on preferences by eliciting their true preference on nicotine.

19 When amount dummies and delay dummies are used as regressors instead of the corresponding variables, similar results are obtained. The results for the money session are not presented, since the estimation routine did not converge. 
We unequivocally confirmed that smokers are more impatient than non-smokers in the money, hypothetical tobacco, and real tobacco sessions, which is consistent with previous studies. On the other hand, short-term nicotine deprivation makes smokers even more impatient. This latter result is obtained only in the real tobacco session, where subjects actually consume their tobacco rewards at the specified time. This suggests that giving appropriate incentives is crucial for the elicitation of true preferences when smoking is involved. When the sample is restricted to heavy smokers, the effect is even stronger.

Overall, these results suggest that nicotine concentration has different effects in the short-run than in the long-run; although long-term intake of nicotine, which implies higher nicotine concentrations over time, makes people more impatient, short-term nicotine deprivation, which causes a lower nicotine concentration, makes smokers even more impatient. In other words, nicotine intake has different effects on the time preferences of addicted and non-addicted subjects. ${ }^{20}$ Investigation of the neurological basis for these effects remains as a target for future research.

${ }^{20}$ However, an alternative hypothesis exists. Long-term smokers may simply experience the repeated frequent occurrence of short-term deprivation, and thus become impatient. In this case, the long-term and short-term effects of nicotine would be due to the same phenomenon. 


\section{References}

Badger, G.J., Bickel, W.K., Giordano, L.A., Jacobs, E.A., Loewenstein, G., Marsch, L., 2007. Altered states: The impact of immediate craving on the valuation of current and future opioids. Journal of Health Economics 26, 865-876.

Baker F., Johnson M.W., Bickel W.K., 2003. Delay discounting in current and never-before cigarette smokers: similarities and differences across commodity, sign, and magnitude. Journal of Abnormal Psychology 112, 382-392.

Benzion, U., Rapoport, A., Yagil, J., 1989. Discount rates inferred from decisions: An experimental study, Management Science 35, 270-284.

Bickel, W.K., Odum, A.L., Madden, G.J., 1999. Impulsivity and cigarette smoking: Delay discounting in current, never, and exsmokers. Psychopharmacology 146, 447-454.

Dallery, J, Locey, M.L., 2005. Effects of acute and chronic nicotine on impulsive choice in rats. Behavioural Pharmacology16(1), 15-23.

Dallery, J., Raiff, B.R., 2007. Delay discounting predicts cigarette smoking in a laboratory model of abstinence reinforcement, Psychopharmacology 190, 485-496.

Field, M., Santarcangelo, M., Sumnall, H., Goudie, A., Cole, J., 2006. Delay discounting and the behavioural economics of cigarette purchases in smokers: the effects of nicotine deprivation. Psychopharmacology186, 255-263

Frederick, S., Loewenstein, G., O’Donoghue, T., 2002. Time discounting and time preference: A critical review. Journal of Economic Literature 40, 351-401.

Giordano, L.A., Bickel, W.K., Loewenstein, G., Jacobs, E.A., Marsch, L.A., Badger, G.J., 2002. Mild opioid deprivation increases the degree that opioid-dependent outpatients discount delayed heroin and money. Psychopharmacology 163 (2), 174-182. 
Ida, T., Goto, R., Takahashi, Y., Nishimura, S., 2011. Can economic-psychological parameters predict successful smoking cessation? Journal of Socio-Economics 40(3), 285-295.

Khwaja, A., Silverman, D., Sloan, F., 2007. Time preference, time discounting, and smoking decisions. Journal of Health Economics 26, 927-949.

Kinari, Y., Ohtake, F., Tsutsui, Y., 2009. Time discounting: declining impatience and interval effect. Journal of Risk and Uncertainty 39, 87-112.

Kirby, K.N., Marakovic, N.N., 1995. Modeling myopic decisions: Evidence for hyperbolic delay-discounting within subjects and amounts. Organizational Behavior and Human Decision Processes 64, 22-30.

Krishnan-Sarin, S., Reynolds, B., Duhig, A.M., Smith, A., Liss, T., McFetridge, A., Cavallo, D.A., Carroll, K.M., Potenza, M.N., 2007. Behavioral impulsivity predicts treatment outcome in a smoking cessation program for adolescent smokers. Drug and Alcohol Dependence 88, 79-82.

Loewenstein, G., Prelec, D., 1992. Anomalies in intertemporal choice: evidence and an interpretation. Quarterly Journal of Economics 107, 573-597.

Madden G.J., Begotka A., Raiff B.R., Kastern, L., 2003. Delay discounting of real and hypothetical rewards. Experimental and Clinical Psychopharmacology 11, 139-145.

Madden, G.J., Raiff, B.R., Lagorio, C.H., Begotka, A., Mueller, A., Hehli, D., Wegener, A., 2004. Delay discounting of potentially real and hypothetical rewards. Part II: between- and within-subject comparisons. Experimental and Clinical Psychopharmacology 12, 251-261.

Mitchell, S.H., 2004. Effects of short-term nicotine deprivation on decision-making: delay, uncertainty, and effort discounting. Nicotine \& Tobacco Research 6, 819-828. 
Myerson, J., Green, L., Warusawitharana, M., 2001. Area under the curve as a measure of discounting. Journal of the Experimental Analysis of Behavior 76, 235-243.

Odum, A.L., Baumann, A.A.L., 2007. Cigarette smokers show steeper discounting of both food and cigarettes than money. Drug and Alcohol Dependence 91, 293-296.

Ohmura, Y., Takahashi, T., Kitamura, N., 2005. Discounting delayed and probabilistic monetary gains and losses by smokers of cigarettes. Psychopharmacology (Berl) 182(4), 508-515.

Reynolds, B., 2004. Do high rates of cigarette consumption increase delay discounting? A cross-sectional comparison of adolescent smokers and young-adult smokers and nonsmokers. Behavioral Processes 67, 545-549.

Reynolds, B., Schiffbauer, R., 2004. Measuring state changes in human delay discounting: an experiential discounting task. Behavioral Processes 67 (3), 343-356.

Reynolds, B., Karraker, K., Horn, K., Richards, J.B., 2003. Delay and probability discounting as related to different stages of adolescent smoking and nonsmoking. Behavioral Processes 64, 333-344.

Reynolds, B., Ortengren, A., Richards, J.B., de Wit, H., 2006. Dimensions of impulsive behavior: personality and behavioral measures. Personality Individual Differences 40, 305-315.

Reynolds, B., Patak, M., Shroff, P., Penfold, R.B., Melanko, S., Duhig, A.M., 2007. Laboratory and self-report assessments of impulsive behavior in adolescent daily smokers and nonsmokers. Experimental and Clinical Psychopharmacology 15, 264-271.

Sasaki, S., Xie, S., Ikeda, S., Qin, J., Tsutsui, Y., 2011. Time discounting: Delay effect and procrastinating behavior, CREED Working Paper, University of Amsterdam.

Sato, M., Ohkusa, Y., 2003. The relationship between smoking initiation and time 
discount factor, risk aversion and information. Applied Economics Letters 10, 287 -289 .

Sayette, M.A., Loewenstein, G., Kirchner, T.R., Travis, T., 2005. Effects of smoking urge on temporal cognition. Psychology of Addictive Behaviors 19(1), 88-93.

Tsutsui-Kimura, I., Ohmura, Y., Izumi, T., Yamaguchi, T., Yoshida, T., Yoshioka, M., 2010. Nicotine provokes impulsive-like action by stimulating alpha4beta2 nicotinic acetylcholine receptors in the infralimbic, but not in the prelimbic cortex. Psychopharmacology (Berl) 209(4), 351-9.

Yoon, J.H., Higgins, S.T., Bradstreet, M.P., Badger, G.J., Thomas, C.S., 2009. Changes in the relative reinforcing effects of cigarette smoking as a function of initial abstinence. Psychopharmacology 205, 305-318. 
Table 1 Questions in real tobacco and hypothetical tobacco sessions

\begin{tabular}{|c|c|c|c|c|c|c|c|c|c|c|}
\hline \multirow{2}{*}{ amount(A) } & \multirow[b]{2}{*}{$0 \%$} & \multicolumn{9}{|c|}{ Rate of return } \\
\hline & & $33.3 \%$ & $50 \%$ & $100 \%$ & $200 \%$ & $300 \%$ & $500 \%$ & $700 \%$ & $1100 \%$ & $1500 \%$ \\
\hline 1puff & 1 puff & & & 2 puffs & & 0.5 & & 1 & 1.5 & 2 \\
\hline 2 puffs & & & & 0.5 & & 1 & 1.5 & 2 & & \\
\hline 0.5 & & & & 1 & 1.5 & & 2 & & & \\
\hline 1 & & & 1.5 & 2 & & & & & & \\
\hline 1.5 & & 2 & & & & & & & & \\
\hline
\end{tabular}

Note: The amounts of the rewards corresponding to option B are shown in each cell. Each number represents a number of cigarettes, unless otherwise mentioned. The length of the period used to determine rates of return is 30 minutes in the real tobacco session and 12 hours in the hypothetical tobacco session. 
Table 2 Sixteen questions asked for each delay in money session

\begin{tabular}{rcccrrr}
\hline \multirow{2}{*}{ amount(A) } & \multicolumn{5}{c}{ Rate of return (annual) } \\
& $0 \%$ & $50 \%$ & $100 \%$ & $150 \%$ & $200 \%$ & $300 \%$ \\
\hline 1000 & 1000 & 1019 & 1039 & 1058 & 1077 & 1116 \\
2000 & & 2039 & 2077 & 2116 & 2154 & 2231 \\
3000 & & 3058 & 3116 & 3174 & 3231 & 3347 \\
\hline
\end{tabular}

Note: The amount of rewards (yen) in option B is shown in each cell. 
Table 3 Estimation results of panel logit regression: smokers vs. non-smokers

\begin{tabular}{|c|c|c|c|c|c|c|}
\hline & Coef. & $\mathrm{p}$ value & Coef. & $p$ value & Coef. & $p$ value \\
\hline Constant & -2.205 & {$[0.000] * *$} & -1.691 & {$[0.000] * *$} & -1.434 & {$[0.000] * *$} \\
\hline D_SMOKER & -0.658 & {$[0.000] * *$} & -0.662 & {$[0.000] * *$} & -0.663 & {$[0.000] * *$} \\
\hline$D E L A Y$ & 0.03 & {$[0.170]$} & & & & \\
\hline AMOUNT & 0.001 & {$[0.000] * *$} & & & & \\
\hline RETURN & 0.01 & {$[0.000] * *$} & 0.01 & {$[0.000] * *$} & & \\
\hline$D_{-} D E L A Y_{-} M 2$ & & & 0.3 & {$[0.003] * *$} & 0.3 & {$[0.003] * *$} \\
\hline$D_{-} D E L A Y_{-} M 3$ & & & 0.285 & {$[0.004] * *$} & 0.285 & {$[0.004] * *$} \\
\hline$D_{-} D E L A Y_{-} M 4$ & & & 0.201 & {$[0.043] *$} & 0.201 & {$[0.043] *$} \\
\hline$D_{-} D E L A Y_{-} M 5$ & & & 0.201 & {$[0.043] *$} & 0.201 & {$[0.043] *$} \\
\hline D_AMOUNT_M2 & & & 0.987 & {$[0.000] * *$} & 1.017 & {$[0.000] * *$} \\
\hline D_AMOUNT_M3 & & & 1.434 & {$[0.000] * *$} & 1.471 & {$[0.000] * *$} \\
\hline D_RETURN_M4 & & & & & 0.84 & {$[0.000] * *$} \\
\hline D_RETURN_M5 & & & & & 1.459 & {$[0.000] * *$} \\
\hline D_RETURN_M6 & & & & & 1.692 & {$[0.000] * *$} \\
\hline D_RETURN_M7 & & & & & 2.594 & {$[0.000] * *$} \\
\hline Pseudo $\mathrm{R}^{2}$ & & 0.184 & & 0.184 & & 0.185 \\
\hline Observation & & 5360 & & 5360 & & 5360 \\
\hline
\end{tabular}

Note: ** indicates significance at the $1 \%$ level and * at the $5 \%$ level. 
Table 4 Estimation results of panel logit regression: effect of deprivation

\begin{tabular}{|c|c|c|c|c|c|c|}
\hline & \multicolumn{2}{|c|}{ Real tobacco session } & \multicolumn{2}{|c|}{ Hypothetical tobacco session } & \multirow{2}{*}{$\begin{array}{l}\text { Money } \\
\text { Coef. }\end{array}$} & \multirow{2}{*}{$\begin{array}{l}\text { session } \\
\mathrm{p} \text { value }\end{array}$} \\
\hline & Coef. & $\mathrm{p}$ value & Coef. & $p$ value & & \\
\hline D_DEPRIVATION & -0.161 & {$[0.006] * *$} & 0.048 & {$[0.411]$} & 0.089 & {$[0.219]$} \\
\hline$D E L A Y$ & -0.031 & {$[0.000] * *$} & -0.0002 & {$[0.951]$} & 0.087 & {$[0.001] * *$} \\
\hline AMOUNT & -1.750 & {$[0.000] * *$} & -1.321 & {$[0.000] * *$} & 0.002 & {$[0.000] * *$} \\
\hline RETURN & 0.002 & {$[0.000] * *$} & 0.002 & {$[0.000] * *$} & 0.021 & {$[0.000] * *$} \\
\hline Pseudo $\mathrm{R}^{2}$ & & 0.208 & & 0.201 & & 0.432 \\
\hline Observation & 49persons & 7840 & 47persons & 7520 & 50persons & 8000 \\
\hline
\end{tabular}

\begin{tabular}{|c|c|c|c|c|c|c|c|}
\hline & \multicolumn{2}{|c|}{ Real tobacco session } & \multicolumn{2}{|c|}{ Hypothetical tobacco session } & & \multicolumn{2}{|c|}{ Money session } \\
\hline & Coef. & $\mathrm{p}$ value & Coef. & $\mathrm{p}$ value & & Coef. & $p$ value \\
\hline D_DEPRIVATION & -0.166 & {$[0.005] * *$} & 0.051 & {$[0.400]$} & D_DEPRIVATION & 0.090 & {$[0.215]$} \\
\hline RETURN & 0.002 & {$[0.000] * *$} & 0.003 & {$[0.000] * *$} & RETURN & 0.022 & {$[0.000] * *$} \\
\hline$D_{-} D E L A Y_{-} T 2$ & -0.113 & {$[0.234]$} & -0.118 & {$[0.216]$} & $D_{-} D E L A Y_{-} M 2$ & 0.637 & {$[0.000] * *$} \\
\hline$D_{-} D E L A Y_{-} T 3$ & -0.185 & {$[0.052]$} & -0.195 & {$[0.041] *$} & $D_{-} D E L A Y_{-} M 3$ & 0.624 & {$[0.000] * *$} \\
\hline$D_{-} D E L A Y_{-} T 4$ & -0.158 & {$[0.096]$} & 0.127 & {$[0.183]$} & $D_{-} D E L A Y_{-} M 4$ & 0.524 & {$[0.000] * *$} \\
\hline$D_{-} D E L A Y_{-} T 5$ & -0.462 & {$[0.000] * *$} & -0.149 & {$[0.117]$} & $D_{-} D E L A Y_{-} M 5$ & 0.498 & {$[0.000] * *$} \\
\hline$D_{-} A M O U N T_{-} T 2$ & 0.552 & {$[0.000] * *$} & 0.753 & {$[0.000] * *$} & D_AMOUNT_M2 & 2.084 & {$[0.000] * *$} \\
\hline$D_{-} A M O U N T_{-} T 3$ & 0.060 & {$[0.472]$} & 0.535 & {$[0.000] * *$} & D_AMOUNT_M3 & 3.120 & {$[0.000] * *$} \\
\hline D_AMOUNT_T4 & -1.684 & {$[0.000] * *$} & -1.280 & {$[0.000] * *$} & & & \\
\hline$D_{-} A M O U N T_{-} T 5$ & -1.854 & {$[0.000] * *$} & -1.368 & {$[0.000] * *$} & & & \\
\hline Pseudo $R^{2}$ & & 0.228 & & 0.234 & Pseudo R2 & & 0.442 \\
\hline Observation & 49persons & 7840 & 47persons & 7520 & Observation & 50persons & 8000 \\
\hline
\end{tabular}

Note: The numbers of observations differ because those who only chose A or only chose B are excluded. 
Table 5 Estimation results of panel logit regression: light vs. heavy smokers

Separation (a)

\begin{tabular}{|c|c|c|c|c|c|c|c|c|}
\hline & \multicolumn{4}{|c|}{ Real tobacco session } & \multicolumn{4}{|c|}{ Hypothetical tobacco session } \\
\hline & \multicolumn{2}{|c|}{ light smokers } & \multicolumn{2}{|c|}{ heavy smokers } & \multicolumn{2}{|c|}{ light smokers } & \multicolumn{2}{|c|}{ heavy smokers } \\
\hline & Coef. & $p$ value & Coef. & $\mathrm{p}$ value & Coef. & $\mathrm{p}$ value & Coef. & $\mathrm{p}$ value \\
\hline D_DEPRIVATION & 0.014 & {$[0.886]$} & -0.391 & {$[0.000] * *$} & -0.161 & {$[0.098]$} & 0.129 & {$[0.089]$} \\
\hline RETURN & 0.002 & {$[0.000] * *$} & 0.003 & {$[0.000] * *$} & 0.002 & {$[0.000] * *$} & 0.003 & {$[0.000] * *$} \\
\hline$D E L A Y$ & -0.017 & {$[0.121]$} & -0.042 & {$[0.000] * *$} & -0.005 & {$[0.401]$} & 0.002 & {$[0.707]$} \\
\hline AMOUNT & -2.311 & {$[0.000] * *$} & -1.46 & {$[0.000] * *$} & -1.634 & {$[0.000] * *$} & -1.075 & {$[0.000] * *$} \\
\hline Pseudo $\mathrm{R}^{2}$ & & 0.210 & & 0.232 & & 0.202 & & 0.214 \\
\hline Observations & 17persons & 2720 & 31 persons & 4960 & 17persons & 2720 & 29persons & 4640 \\
\hline
\end{tabular}

\begin{tabular}{|c|c|c|c|c|c|c|c|c|}
\hline \multicolumn{9}{|c|}{ Separation (b) } \\
\hline & \multicolumn{4}{|c|}{ Real tobacco session } & \multicolumn{4}{|c|}{ Hypothetical tobacco session } \\
\hline & \multicolumn{2}{|c|}{ light smokers } & \multicolumn{2}{|c|}{ heavy smokers } & \multicolumn{2}{|c|}{ light smokers } & \multicolumn{2}{|c|}{ heavy smokers } \\
\hline & Coef. & $p$ value & Coef. & $p$ value & Coef. & $p$ value & Coef. & $p$ value \\
\hline D_DEPRIVATION & 0.058 & {$[0.424]$} & -0.876 & {$[0.000] * *$} & 0.185 & {$[0.010] *$} & -0.363 & {$[0.001] * *$} \\
\hline RETURN & 0.002 & {$[0.000] * *$} & 0.004 & {$[0.000] * *$} & 0.002 & {$[0.000] * *$} & 0.003 & {$[0.000] * *$} \\
\hline$D E L A Y$ & -0.024 & {$[0.005] * *$} & -0.05 & {$[0.000] * *$} & 0.003 & {$[0.524]$} & -0.008 & {$[0.172]$} \\
\hline AMOUNT & -2.114 & {$[0.000] * *$} & -1.169 & {$[0.000] * *$} & -1.557 & {$[0.000] * *$} & -0.736 & {$[0.000] * *$} \\
\hline Pseudo $\mathrm{R}^{2}$ & & 0.218 & & 0.249 & & 0.211 & & 0.215 \\
\hline Observation & 31 persons & 4960 & 17persons & 2720 & 30persons & 4800 & 16persons & 2560 \\
\hline
\end{tabular}

Note: Note: The numbers of observations differ because those who only chose A or only chose B are excluded. 


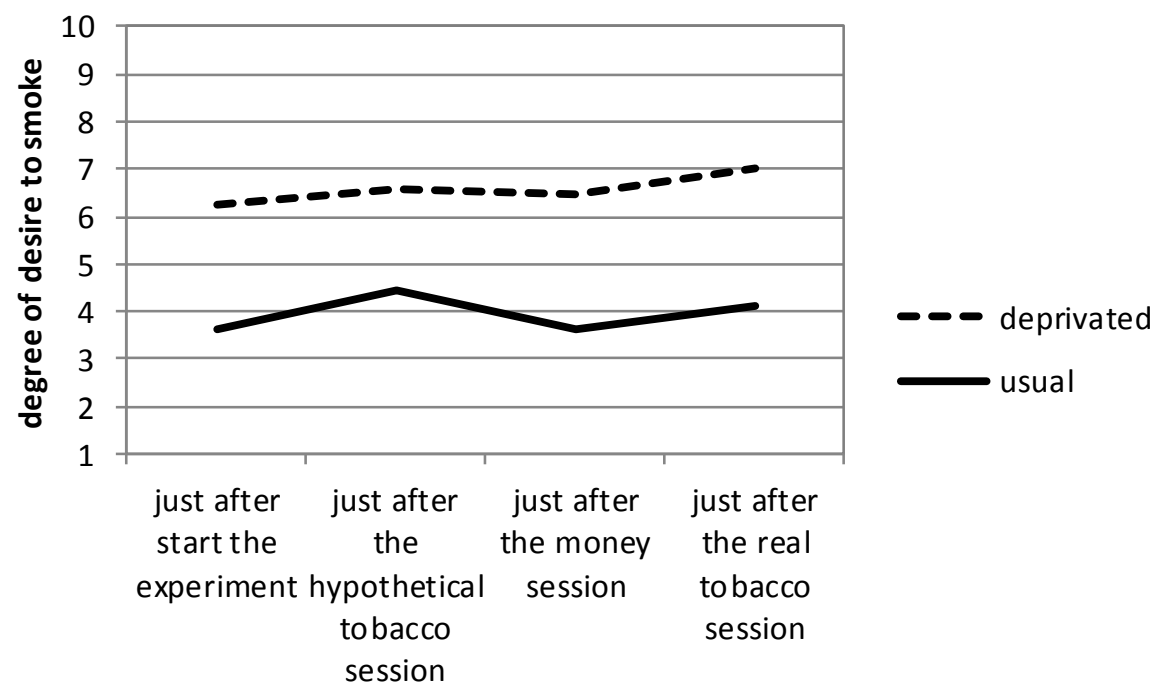

Figure 1 How strongly do you want to smoke now?

Note: 49 subjects are asked to choose from 1 ("I do not want to smoke now") to 10 ("I want very much to smoke now.” They were asked the question twice, so that the number of responses is 98. 


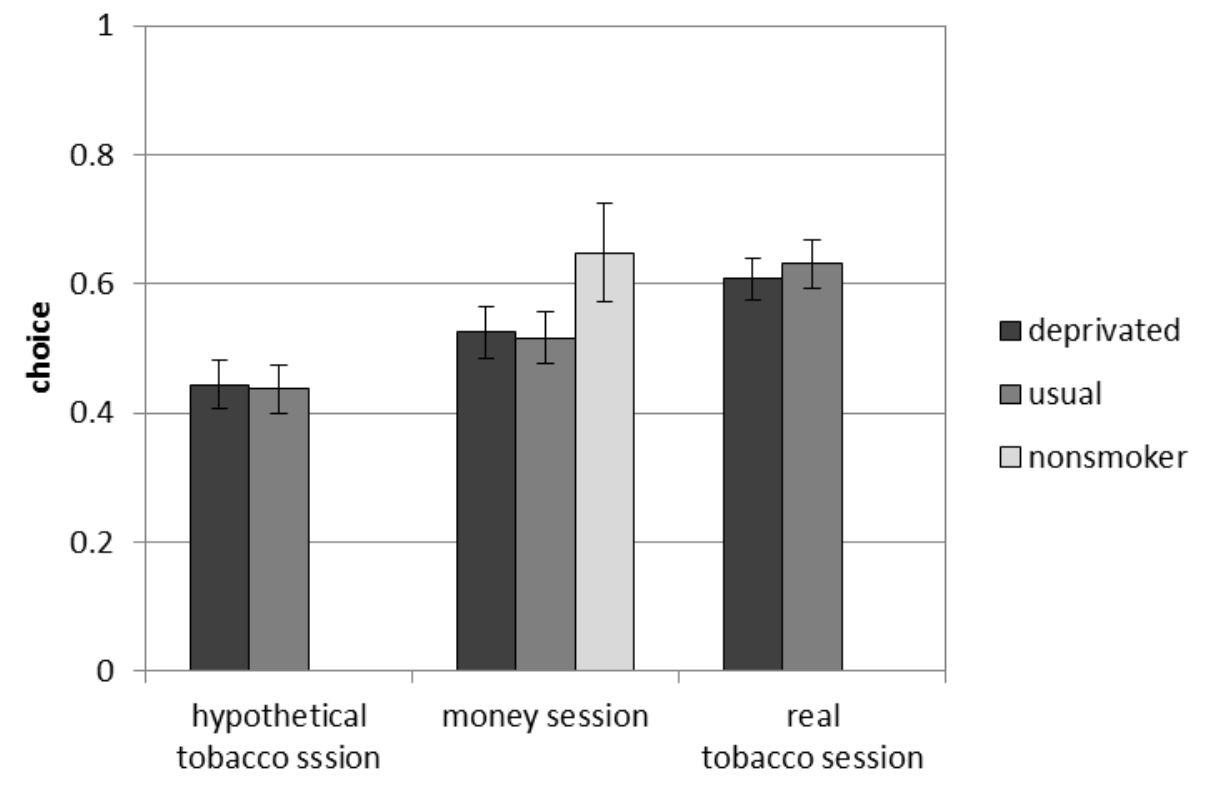

Figure 2 Fraction who chose the later option (B)

Note: Vertical bars represent \pm SEM (standard error of means) 


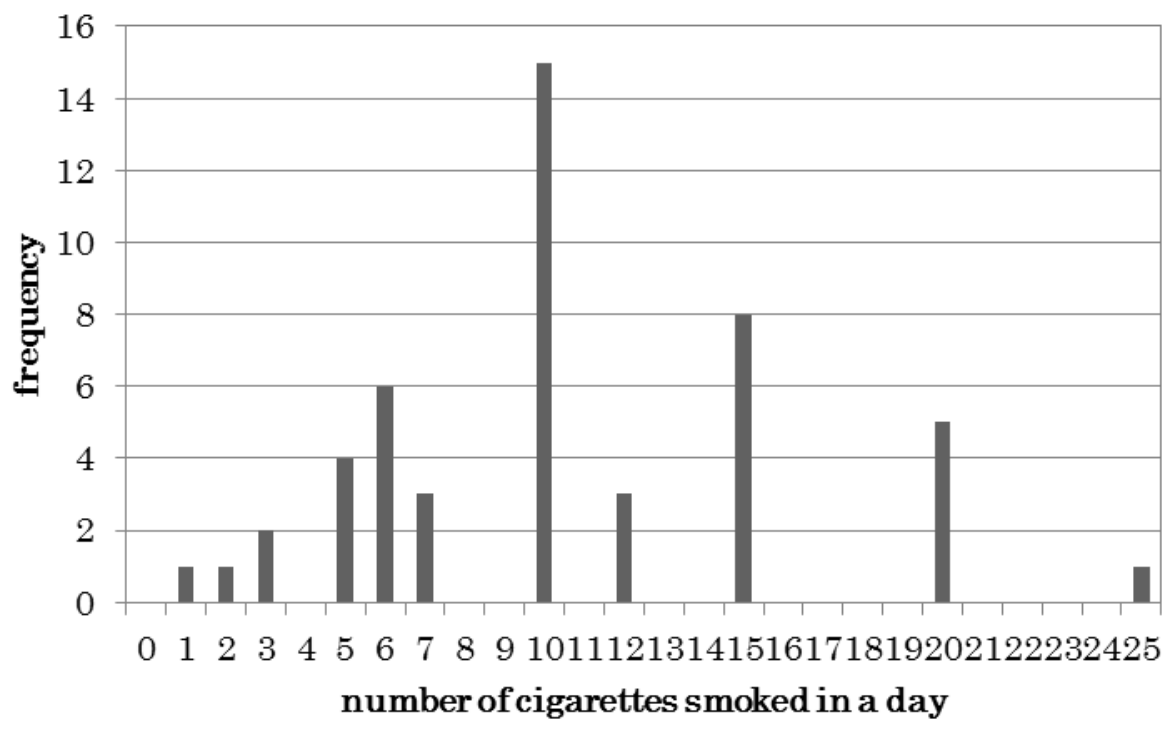

Figure 3 Histogram of the number of cigarettes that subjects typically smoke per day 
Appendix: Definition of variables used in regressions

\begin{tabular}{|c|c|}
\hline variable & Explanation \\
\hline$D_{-} S I$ & smoker dummy variable: 1 when subjects is smoker, 0 otherwise \\
\hline D_DEPRIVATION & $\begin{array}{l}\text { deprivation dummy variable: } 1 \text { when subjects is nicotine deprived, } 0 \\
\text { otherwise }\end{array}$ \\
\hline$D E L A Y$ & time of earlier reward \\
\hline$D_{-} D E L A Y_{-} M 2$ & $\begin{array}{l}\text { delay dummy variable in money session: } 1 \text { when delay is one week, } 0 \\
\text { otherwise }\end{array}$ \\
\hline$D_{-} D E L A Y_{-} M 3$ & $\begin{array}{l}\text { delay dummy variable in money session: } 1 \text { when delay is two weeks, } 0 \\
\text { otherwise }\end{array}$ \\
\hline$D_{-} D E L A Y_{-} M 4$ & $\begin{array}{l}\text { delay dummy variable in money session: } 1 \text { when delay is three weeks, } 0 \\
\text { otherwise }\end{array}$ \\
\hline$D_{-} D E L A Y_{-} M 5$ & $\begin{array}{l}\text { delay dummy variable in money session: } 1 \text { when delay is four weeks, } 0 \\
\text { otherwise }\end{array}$ \\
\hline$D_{-} D$ & $\begin{array}{l}\text { delay dummy variable in tobacco sessions: } 1 \text { when delay is } 10 \mathrm{~min}, 0 \\
\text { otherwise in real tobacco session, } 1 \text { when delay is } 1 \text { hours, } 0 \text { otherwise in } \\
\text { hypothetical tobacco session }\end{array}$ \\
\hline$D_{-} D E L A Y_{-} T 3$ & $\begin{array}{l}\text { delay dummy variable in tobacco sessions: } 1 \text { when delay is } 20 \mathrm{~min}, 0 \\
\text { otherwise in real tobacco session, } 1 \text { when delay is } 3 \text { hours, } 0 \text { otherwise in } \\
\text { hypothetical tobacco session }\end{array}$ \\
\hline$D_{-} D E L A Y_{-} T 4$ & $\begin{array}{l}\text { delay dummy variable in tobacco sessions: } 1 \text { when delay is } 30 \mathrm{~min}, 0 \\
\text { otherwise in real tobacco session, } 1 \text { when delay is } 12 \text { hours, } 0 \text { otherwise } \\
\text { in hypothetical tobacco session }\end{array}$ \\
\hline$D_{-} D E L A Y_{-} T 5$ & $\begin{array}{l}\text { delay dummy variable in tobacco sessions: } 1 \text { when delay is } 40 \mathrm{~min}, 0 \\
\text { otherwise in real tobacco session, } 1 \text { when delay is } 24 \text { hours, } 0 \text { otherwise } \\
\text { in hypothetical tobacco session }\end{array}$ \\
\hline AMOUNT & \\
\hline$D_{-} A M O U N T_{-} M 2$ & $\begin{array}{l}\text { delay amount variable in money session: } 1 \text { when amount is } 2000 \text { yen, } 0 \\
\text { otherwise }\end{array}$ \\
\hline$D_{-} A M O U N T_{-} M 3$ & $\begin{array}{l}\text { delay amount variable in money session: } 1 \text { when amount is } 3000 \text { yen, } 0 \\
\text { otherwise }\end{array}$ \\
\hline$D_{-} A M O U N T_{-} T 2$ & $\begin{array}{l}\text { delay amount variable in tobacco sessions: } 1 \text { when amount is } 2 \text { puffs, } 0 \\
\text { otherwise }\end{array}$ \\
\hline$D_{-} A M O U N T_{-} T 3$ & $\begin{array}{l}\text { delay amount variable in tobacco sessions: } 1 \text { when amount is } 0.5 \\
\text { cigarettes, } 0 \text { otherwise }\end{array}$ \\
\hline
\end{tabular}


D_AMOUNT_T4 delay amount variable in tobacco sessions: 1 when amount is 1 cigarettes, 0 otherwise

D_AMOUNT_T5 delay amount variable in tobacco sessions: 1 when amount is 1.5 cigarettes, 0 otherwise

RETURN return of later reward

D_RETURN_M4 delay return variable in money session: 1 when return is $100 \%, 0$ otherwise

D_RETURN_M5 delay return variable in money session: 1 when return is $150 \%, 0$ otherwise

D_RETURN_M6 delay return variable in money session: 1 when return is $200 \%, 0$ otherwise

D_RETURN_M7 delay return variable in money session: 1 when return is $300 \%, 0$ otherwise 
